\title{
LOCALIZED INDUCTION HIERARCHY AND WEINGARTEN SYSTEMS
}

\author{
Ron PERLine \\ Dept. of Mathematics and Computer Science, Drexel University \\ AbStract. We describe a method of constructing Weingarten systems of triply \\ orthogonal coordinates, related to the localized induction equation hierarchy of \\ integrable geometric evolution equations.
}

\author{
Submitted to \\ Physics Letters A
}

PACS numbers: 03.40.Gc, 02.40.+m, 11.10.Lm, 68.10-m 


\section{Introduction.}

Recently, there has been a great deal of research related to problems from classical differential geometry, approached from the modern perspective of integrable systems (or soliton theory). As representative works we mention those of Sym, Pinkall, Bobenko, and Melko and Sterling [Sym], [Bur], [Bob], [Mel-S].

In a related series of papers [Lan-P 1-5], [Per], we have studied the localized induction equation (LIE) and have investigated its role in differential geometry. LIE is a local geometric evolution equation defined on space curves via the equation

$$
\gamma_{t}=\gamma_{s} \times \gamma_{s s}
$$

where $s$ is arclength parameter for an evolving space curve $\gamma(s, t) \in R^{3}$. LIE can also be written as $\gamma_{t}=\kappa B$, where $\kappa$ is the curvature and $B$ is the binormal of the curve. LIE is an extremely idealized model for the evolution of the centerline of a thin, isolated vortex tube in an inviscid fluid; it was first developed at the turn of the century by da Rios, a student of the geometer Levi-Civita, and rediscovered some thirty years ago (for derivation and history, see [Bat],[Lam 1],[Ric 1]). Thus the roots of LIE are historically grounded in the boundary between mathematical physics and geometry.

Hasimoto [Has] found the connection between LIE and soliton theory: if $\gamma$ evolves according to LIE, then Hasimoto showed that the asso-

ciated complex curvature function $\psi(s)=\kappa(s) e^{i \int^{s} \tau(u) d u}(\tau$ is the curve's torsion) evolves according to the cubic nonlinear Schrödinger equation (NLS) $\psi_{t}=i\left(\psi_{s s}+\frac{1}{2}|\psi|^{2} \psi\right)$. NLS is one of the standard examples of an integrable partial differential equation; the result of Hasimoto states that LIE is a geometric realization of NLS. We remark that there is now machinery for producing geometric realizations of integrable equations via the soliton surface approach [Sym]. In particular, This method indeed "produces" LIE as a geometric realization of NLS.

As a consequence of the LIE-NLS corresondence, LIE inherits the characteristics associated with integrable equations: soliton solutions, an infinite 
sequence of conserved Hamiltonians in involution, and a corresponding hierarchy of commuting Hamiltonian vector fields. We refer to this hierarchy as the localized induction hierarchy (LIH).

As a curve evolves through space with a velocity field which is a member of LIH, it sweeps out a surface; and one can ask if there is any interesting structure to the resulting surface. For example, if the curve $\gamma(s, t)$ evolves according to LIE, then it is easy to see that $\gamma\left(s, t_{0}\right)$ is a geodesic on the resulting surface, for any fixed time $t_{0}$.

In a similar fashion, we have recently constructed pseudospherical surfaces (= surface of constant negative Gauss curvature) using the integrability properties of LIH [Per]. We demonstrated that certain soliton curves (= critical points for linear combinations of the conserved Hamiltonians), evolving according to linear combinations of the vector fields from LIH, sweep out pseudospherical surfaces.

In this paper, we extend this result to give a dynamical prescription for producing Weingarten systems. A Weingarten system is a triply orthogonal system of surfaces, such that the surfaces in one family are pseudospherical [Eis]. The subject of triply orthogonal systems is an old one in mathematical physics and geometry, which continues to have interesting developments [Mil]. The connection we make between Weingarten systems and integrable geometric evolution equations seems to be new.

We begin by reviewing relevant facts about the structure of LIH and related vector field hierarchies needed for our construction. We then describe the procedure for constructing Weingarten systems. To keep the paper selfcontained, we briefly include some material from an earlier paper. 


\section{LIH and related hierarchies.}

As stated in the introduction, LIE belongs to an infinite hierarchy of commuting evolution equations on curves, all of the form $\gamma_{t}=X_{n}=a T+$ $b N+c B$, where $\{T, N, B\}$ is the Frenet frame along the curve, and $a, b, c$ are functions (polynomial) of $\kappa, \tau, \kappa^{\prime}=\kappa_{s}, \tau^{\prime}=\tau_{s}$, and higher derivatives with respect to $s$. We list the first few terms of the hierarchy, as well as their associated Hamiltonians:

$$
\begin{aligned}
& X_{1}=\kappa B, \quad I_{1}=\int_{\gamma} d s, \\
& X_{2}=\frac{\kappa^{2}}{2} T+\kappa^{\prime} N+\kappa \tau B, \quad I_{2}=\int_{\gamma}-\tau d s, \\
& X_{3}=\kappa^{2} \tau T+\left(2 \kappa^{\prime} \tau+\kappa \tau^{\prime}\right) N+\left(\kappa \tau^{2}-\kappa^{\prime \prime}-\frac{1}{2} \kappa^{3}\right) B, \\
& I_{3}=\int_{\gamma} \frac{1}{2} \kappa^{2} d s, \\
& X_{4}=\left(-\kappa \kappa^{\prime \prime}+\frac{1}{2}\left(\kappa^{\prime}\right)^{2}+\frac{3}{2} \kappa^{2} \tau^{2}-\frac{3}{8} \kappa^{4}\right) T \\
&+\left(-\kappa^{\prime \prime \prime}+3 \kappa \tau \tau^{\prime}+3 \kappa^{\prime} \tau^{2}-\frac{3}{2} \kappa^{2} \kappa^{\prime}\right) N \\
&+\left(\kappa \tau^{3}-3\left(\kappa^{\prime} \tau\right)^{\prime}-\frac{3}{2} \kappa^{3} \tau-\kappa \tau^{\prime \prime}\right) B, \\
& I_{4}=\int_{\gamma} \frac{1}{2} \kappa^{2} \tau d s,
\end{aligned}
$$

The vector fields of LIH are locally arclength preserving : a vector field $W$ is locally archlength preserving if every segment of a curve $\gamma$ has its length remain constant as $\gamma$ evolves via $\gamma_{t}=W$. Equivalently, $\left\langle W_{s}, T\right\rangle=0$. A discussion of the physical interpretation of some of these vector fields and associated functionals can be found in [Ric 2].

LIH is generated by a recursion operator $X_{n+1}=\mathcal{R} X_{n}, n \geq 0$; if $X=a T+b N+c B$ then $\mathcal{R}(X)=-\mathcal{P}\left(T \times X^{\prime}\right)$, where $\mathcal{P}$ is a parameterization operator $\mathcal{P}(X)=\int^{s}(\kappa b) d s T+b N+c B$. Using $\mathcal{R}$, we can compactly express the first-order variations in curvature and torsion along any vector field $W$ which is locally arclength preserving [Lan-P 3]:

$$
W(\kappa)=<-\mathcal{R}^{2}(W), N>,
$$




$$
W(\tau)=<-\mathcal{R}^{2}(W), B / \kappa>^{\prime}
$$

There are a number of hierarchies of integrable geometric evolution equations, related to LIE, which have interesting geometric properties. These are discussed in more detail in [Lan-P 3]. We recall two which are relevant to our construction of Weingarten systems:

(1) Constant torsion preserving: For $n \geq 0$, the vector fields

$$
Z_{n}=\sum_{k=0}^{2 n-1}\left(\begin{array}{c}
2 n+1 \\
k
\end{array}\right)\left(-\tau_{0}\right)^{k} X_{2 n-k}
$$

preserve the constant torsion condition $\tau=\tau_{0}$. If a constant torsion curve $\gamma$ evolves according to $\gamma_{t}=Z_{n}$, the induced evolution on curvature $\kappa_{t}=Z_{n}(\kappa)$ is the corresponding element of the $(\mathrm{mKdV})$ hierarchy; in particular, $Z_{1}$ induces the $(\mathrm{mKdV})$ evolution $\kappa_{t}=\kappa_{s s s}+\frac{3}{2} \kappa^{2} \kappa_{s}$, recovering a result of Lamb [Lam 2]. (2) Torsion independent: The vector fields

$$
A_{n}=\sum_{k=0}^{n-1}\left(\begin{array}{c}
n-1 \\
k
\end{array}\right)\left(-\tau_{0}\right)^{k} X_{n-k}, \quad n \geq 1
$$

have the property that, along curves $\gamma$ with $\tau=\tau_{0}$, the coefficients of $A_{n}=$ $a T+b N+c B$ have no explicit $\tau$ dependence. The odd vector fields in the sequence are purely binormal; the even vector fields, on the other hand, have zero binormal component. We thus refer to the odd fields as "binormal" and the even fields as "planar-like" (since for planar curves they are tangent to the ambient plane).

\section{Planar-like soliton curves and their Killing fields}

We define a planar-like $n$-soliton curve to be a curve $\gamma$ such that $\gamma$ has constant torsion $\tau=\tau_{0}$ and the vectorfield $\sum_{i=0}^{n} a_{i} A_{2 i+1}$ vanishes along $\gamma$, for some choice of $a_{0}, a_{1}, \ldots, a_{n}$. By scaling the $a_{i}$ 's, we can assume without loss of generality that $a_{n}=1$. The condition $\sum_{i=0}^{n} a_{i} A_{2 i+1}=0$ is a nonlinear differential equation for the curvature $\kappa(s)$ whose coefficients are independent of $\tau$; thus, planar-like soliton curves have the same curvature function as some planar curve, and their torsion differs by a constant $\tau_{0}$ (hence the terminology 
"planar-like"). It is quite easy to show that the condition $\sum_{i=0}^{n} a_{i} A_{2 i+1}=0$ is equivalent to $\gamma$ being a critical point for an appropriate linear combination of the conserved Hamiltonians $I_{i}$.

Previously [Lan-P 2], we have considered the reconstruction problem for certain soliton curves: given the curvature and torsion function for such a curve, is there an efficient way of integrating the associated Frenet equations for the curve? It turns out that a key ingredient is that one can express certain Killing fields in terms of the local geometric invariants of the soliton curve. A Killing field is an infinitesimal isometry; that is, it generates a one-parameter flow of rigid motions. A vector field $V$ is called Killing along $\gamma$ if it extends to a Killing field in $R^{3}$ (modulo translation along the curve). Although we do not reconsider the reconstruction problem here, it will be useful to have formulas for the Killing fields of our planar-like soliton curves:

Proposition: let $\gamma$ be a planar-like soliton curves as defined above. Then (i) The vector field $K_{1}=\sum_{i=1}^{n} a_{i} Z_{i}$ is Killing along $\gamma$.

(ii) The condition $\sum_{i=0}^{n} a_{i} A_{2 i+1}=0$ can be rewritten $\sum_{j=1}^{2 n+1} b_{j} X_{j}=0$, where $b_{j}=\sum_{i=\left[\frac{j-1}{2}\right]}^{n} a_{i}\left(\begin{array}{c}2 i \\ j-1\end{array}\right)\left(-\tau_{0}\right)^{2 i+1-j}$. The vector field $K_{2}=\sum_{j=3}^{2 n+1} b_{j} X_{j-2}$ is Killing along $\gamma$.

Using the definition of a planar-like soliton curve, and the variation formulas for $\kappa$ and $\tau$ given above, it is easy to see that the first order variations of $\tau$ and $\kappa$ in the direction of the fields $K_{1}, K_{2}$ are 0 and $\alpha \kappa^{\prime}(s)$ respectively, where $\alpha$ is some constant. That the variations in $\tau$ and $\kappa$ be of this form is obviously a necessary condition for the fields $K_{1}, K_{2}$ to be Killing along $\gamma$; an argument of Langer and Singer [Lan-S] shows that it is in fact sufficient.

\section{Vector fields associated to a planar-like soliton curve.}

We now state the technical proposition which allows us to construct Weingarten systems:

Proposition: Let $\gamma$ be a planar-like soliton curve. Then:

(i) The planar-like vector field $\mathcal{F}=T(x)+\left(-1 / a_{0}\right)\left(\sum_{i=0}^{n} a_{i} A_{2 i}\right)$ is constant torsion preserving along $\gamma . \mathcal{F}$ is a unit vector field which asymptotically $(s \rightarrow$ $\pm \infty)$ equals $T(x)$. 
(ii) The binormal vector field $\mathcal{G}=\sum_{i=0}^{n-1} e_{i} A_{2 i+1}$ is constant torsion preserving along $\gamma$, where $e_{i}=\sum_{k=0}^{n-i-1} a_{k+i+1}(-1)^{k} \tau_{0}{ }^{2 k}$.

The vector field $\mathcal{F}$ is the same as the vector field $T^{*}$ introduced in [Per]. As stated there, to show $\mathcal{F}$ is constant torsion preserving, it suffices to prove the identity

$$
\mathcal{F}=T(x)+\frac{-1}{a_{0} \tau_{0}^{2}} \sum_{k=0}^{n+1}\left(a_{k-1}-2 a_{k} \tau_{0}^{2}+a_{k+1} \tau_{0}^{4}\right) Z_{k}
$$

along $\gamma$, thus expressing $\mathcal{F}$ as a linear combination of vector fields known to preserve the constant torsion condition.

Similarly, to prove that $\mathcal{G}$ is constant torsion preserving, one simply verifies the identity $\mathcal{G}=K_{2}+\sum_{j=1}^{n-1} g_{j} Z_{j}$, where

$$
g_{j}=2 \sum_{i=0}^{n-j-1} a_{n-i}(-1)^{i+j} \tau_{0}^{2 n-2 i-2 j-1} .
$$

The vector fields $Z_{j}$ are constant torsion preserving along any curve with $\tau=\tau_{0}$; and since $K_{2}$ is Killing, it also is constant torsion preserving along $\gamma$.

5. Weingarten systems. We are now in a position to construct Weingarten systems in a simple manner. Start with a planar-like $n$-soliton curve $\gamma_{0}(s)$. Consider the evolution equations $\gamma_{t_{1}}=\mathcal{F}, \gamma_{t_{2}}=\mathcal{G}$, both with initial conditions $\gamma(0, s)=\gamma_{0}(s)$. The resulting flows commute, and both are locally arc-length preserving. Thus it makes sense to discuss $\gamma\left(s, t_{1}, t_{2}\right)$ which generically (away from self-intersections) defines a coordinate system for some subset $U$ of $R^{3}$; here, $\gamma\left(s, t_{1}, t_{2}\right)$ denotes the image of the initial curve $\gamma_{0}$ after evolving $t_{1}$ time units via the first evolution equation, and $t_{2}$ time units via the second.

As discussed above, $\gamma_{0}$ is a critical curve for an appropriate linear combination of the conserved functionals for LIH. The set of such critical curves is conserved by the evolutions associated with the vector fields $\mathcal{F}, \mathcal{G}$, so for any $t_{1}, t_{2}$ the curve $\gamma\left(s, t_{1}, t_{2}\right)$ is a critical curve. Similarly, the constant torsion condition $\tau=\tau_{0}$ is preserved, so $\gamma\left(s, t_{1}, t_{2}\right)$ is an planar-like soliton curve for all "times" $t_{1}, t_{2}$. 
At a generic point in the set $U$, we have the three tangent vectors $\frac{\partial}{\partial s}=$ $T, \frac{\partial}{\partial t_{1}}=\mathcal{F}, \frac{\partial}{\partial t_{2}}=\mathcal{G}$. By construction, $\mathcal{G}$, which is binormal, is perpendicular to the planar-like field $\mathcal{F}$ and the unit tangent field $T$.

For fixed $t_{2}$, we can refer to the results of [Per] to conclude that the level surface $\gamma\left(s, t_{1}, t_{2}\right)$ is pseudospherical; in fact, $s, t_{1}$ are just the asymptotic coordinates for the pseudospherical surface. Finally, we can construct orthogonal coordinates for the pseudospherical surface by using the principal curvature coordinates $r_{1}=\left(s+t_{1}\right) / 2, r_{2}=\left(s-t_{1}\right) / 2$. It is clear that the coordinate system $\left\{r_{1}, r_{2}, t_{2}\right\}$ is a Weingarten system.

As an example of our construction, we give explicitly the associated one-soliton Weingarten system. We start with the well-known Hasimoto filament [Has]:

$$
\begin{gathered}
x=s-\frac{2 \nu \tanh (\nu s)}{\nu^{2}+\tau_{0}^{2}}, y=\frac{2 \nu \operatorname{sech}(\nu s) \cos \left(\tau_{0} s\right)}{\nu^{2}+\tau_{0}^{2}}, \\
z=\frac{2 \nu \operatorname{sech}(\nu s) \sin \left(\tau_{0} s\right)}{\nu^{2}+\tau_{0}^{2}} .
\end{gathered}
$$

This curve has constant torsion $\tau_{0}$ and curvature $\kappa(s)=2 \nu \operatorname{sech}(\nu s)$, and is critical for the functional $I_{3}-2 \tau_{0} I_{2}+\left(\tau_{0}^{2}+\nu^{2}\right) I_{1}$. For this curve, the vector fields $\mathcal{F}$ and $\mathcal{G}$ restrict to Killing fields, which facilitates the integration of the associated flows. The resulting Weingarten system is given by:

$$
\begin{gathered}
x=\frac{\nu^{2} \cosh \left(s_{1}\right) r_{1}+\cosh \left(s_{1}\right) \tau_{0}^{2} r_{1}-2 \nu \sinh \left(s_{1}\right)}{\left(\nu^{2}+\tau_{0}^{2}\right) \cosh \left(s_{1}\right)}, y=\frac{2 \nu\left(\cos \left(s_{2}\right)\right)}{\left(\nu^{2}+\tau_{0}^{2}\right) \cosh \left(s_{1}\right)}, \\
z=\frac{2 \nu\left(\sin \left(s_{2}\right)\right)}{\left(\nu^{2}+\tau_{0}^{2}\right) \cosh \left(s_{1}\right)},
\end{gathered}
$$

where

$$
s_{1}=\frac{\left(\frac{r_{1}}{2}+\frac{r_{2}}{2}\right) \nu^{2}-2 \tau_{0} t_{2} \nu^{2}+\left(\frac{r_{1}}{2}-\frac{r_{2}}{2}\right) \tau_{0}^{2}}{\nu}, s_{2}=\nu^{2} t_{2}+\tau_{0} r_{2}-t_{2} \tau_{0}^{2}
$$

the image of the Weingarten system is the interior of a cylinder in $R^{3}$.

By our construction, the surfaces corresponding to $t_{2}=$ constant are pseudospherical, and this can be verified directly from the formulas for 
the Weingarten system; their curvature is $-\tau_{0}^{2}$. The other families of surfaces for the Weingarten system also have interesting properties: the surfaces $r_{1}=$ constant are spheres, with curvature $\frac{1}{4} \frac{\left(\nu^{2}+\tau_{0}^{2}\right)^{2}}{\nu^{2}}$, and the surfaces $r_{2}=$ constant are again pseudospherical, with curvature $-\frac{1}{4} \frac{\left(\nu^{2}-\tau_{0}^{2}\right)^{2}}{\nu^{2}}$ Our illustration on the next page shows intersecting pieces of three orthogonal surfaces from our families, corresponding to the parameter values $\nu=2, \tau_{0}=1$. 


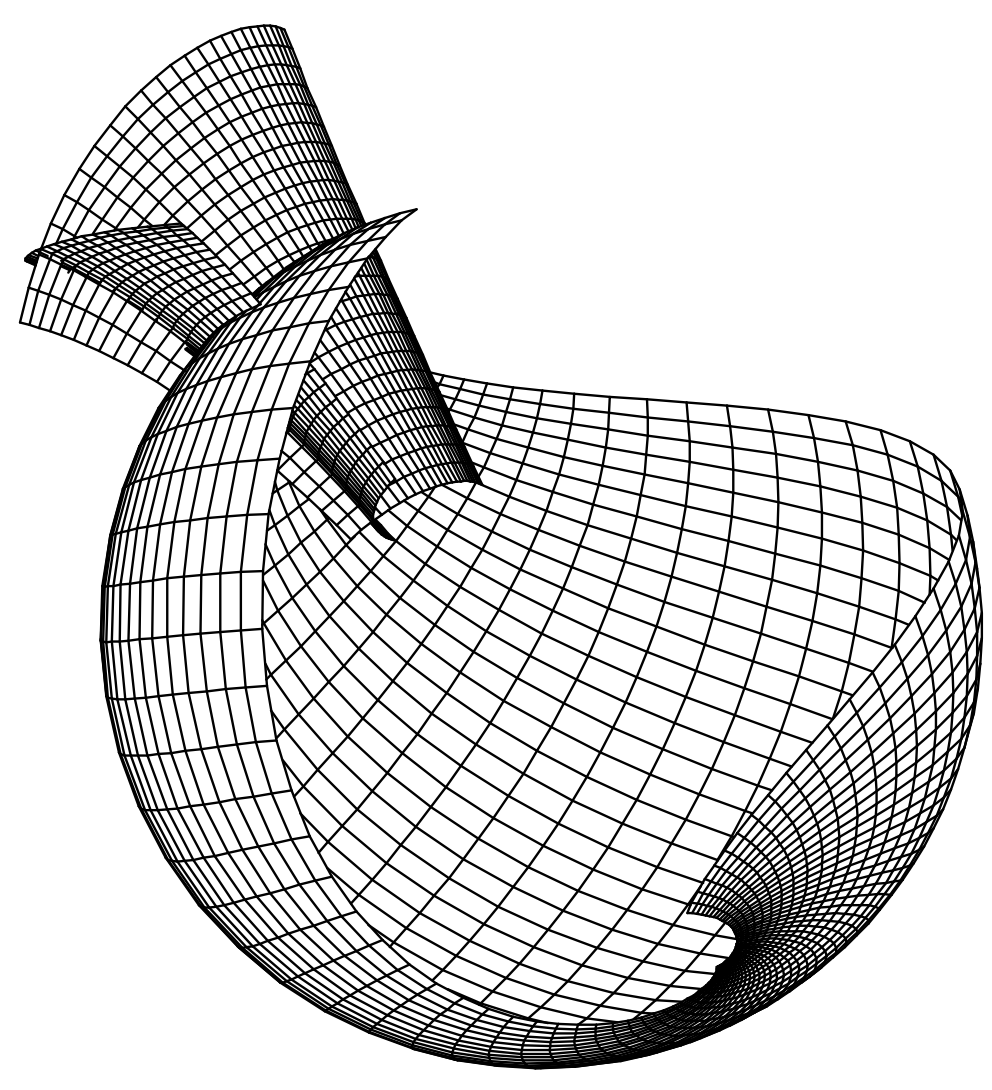


Acknowledgements. The connection of Weingarten systems to integrable systems was brought to our attention by Colin Rogers, whom we thank for several informative and entertaining conversations. Independently, Rogers is developing an alternative approach to the integrability theory of Weingarten systems (private communication), which is likely to be more computational effective than the method described here. The catalyst for these discussions was the recent "Bianchi Days" Workshop of the Warsaw University Physics Department; we extend a warm thanks to Antoni Sym and his colleagues for the interesting and stimulating program they organized.

\section{REFERENCES}

[Bat] G.K. Batchelor, An introduction to fluid dynamics, Cambridge University Press, New York, 1967.

[Bob] A. Bobenko, Functional analysis and its applications 24 (3) (1990), 68.

[Bur $\quad$ F. Burstall, D. Ferus, F. Pedit, U. Pinkall, Annals of Mathematics 138, 173.

[Eis] L. Eisenhart, A treatise of the differential geometry of curves and surfaces, Ginn, Boston, 1909.

[Has] H. Hasimoto, J. Phys. Soc. Japan 31(1) (1971), 293.

[Lam 1] G.L. Lamb, Elements of soliton theory, Wiley Interscience, New York, 1980.

[Lam 2] _ J. Math. Phys. 18 (1977), 1654.

[Lan-P 1] J. Langer and R. Perline, Appl. Math. Lett. 3(2) (1990), 61.

[Lan-P 2] _ J. Nonlinear Sci. 1 (1991), 71.

[Lan-P 3 $]$ _ Fields Institute Proceedings, Mechanics Days, June 1992.

[Lan-P 4] _ Fields Institute Proceedings, Mechanics Days, June 1992.

[Lan-P 5] _ _ Journal of Mathematical Physics 35 (1994), 1732.

[Lan-S] J. Langer and D. Singer, J. Diff. Geom 20 (1984), 1.

[Mel-S] M. Melko and I. Sterling, Ann. of Glob. Anal. and Geom. 11 (1993), 65. 
[Mil] W. Miller, Jr., Symmetry and separation of variables, Addison-Wesley, Reading, Mass., 1977.

[Per] R. Perline, Journal of Physics A 27 (1994), 5335.

[Ric 1] R. Ricca, Nature 352 (1991), 561.

[Ric 2] 는 Phys. Fluids A 4(5) (1992), 938.

[Sym] A. Sym, Geometrical aspects of the Einstein equations and integrable systems, vol. 239, Lecture Notes in Physics, 1985, p. 154. 